\title{
Approximation to General Economic Costs of Mental Illness
}

\author{
Benedikt Frank
}

Heimeranstr. 39, 80339 Munich, Germany

\begin{abstract}
This paper shows characteristics of mental illness and its economic impact, as well as various cost-estimating approaches and insights in missed economic opportunities. To assess the burden of economic diseases, there are three different ways: the human capital, the economic growth and the value of statistical life approach. The first focuses on indirect and direct costs. Direct costs are frequently associated with care and recovery, indirect costs apply to the "invisible costs" correlated with revenue loss due to death, injury, treatment and a fading productivity. The global financial costs of mental illness were evaluated at US\$2.5 trillion, with a significantly bigger impact of indirect costs. Moreover, the effect of mental illness on economic development can only be approximated implicitly. Thus, the lack of production is primary estimated for somatic conditions compared to their corresponding quantity of disabilityadjusted life years (DALYs). According to some research, the total economic productivity drop associated with mental illnesses between 2011 and 2030 is estimated to be US\$16.3 trillion globally. Furthermore, the value of statistical life (VSL) method suggests that trade-offs between risks and capital should be used to assess the probability of injury or death due to psychiatric illness. This computation, like the economic increase effect, is equivalent to that of cardiovascular disease and bigger than that of chronic respiratory disease, cancer, and diabetes. However, greater activism is required to better the existing condition. This involves a shift in thinking away from stigmatizing mentally ill people and supports the activation of financial resources.
\end{abstract}

Keywords: economic costs; economic growth; human capital; mental illness; value of statistical life 